Original Paper

\title{
A Randomized Controlled Trial Comparing Packing with Nonpacking of Perianal Abscess Cavity
}

\author{
Islam $\mathrm{MT}^{1}$, Rahman $\mathrm{MM}^{2}$, Begum $\mathrm{N}^{3}$
}

\begin{abstract}
Introduction: Anorectal abscess is a potentially debilitating condition and one of the common anorectal conditions encountered in practice. Anorectal abscesses are defined by the anatomic space in which they develop and are more common in the perianal and ischiorectal spaces. Traditional management of perianal abscess involves early incision, drainage, curettage and packing of the residual cavity. Cavity packing and dressings are problematic in that they cause significant discomfort and require multiple visits to change the packing until healing. An alternative to the traditional approach is to perform adequate incision and drainage and then to allow healing without the use of cavity dressings.
\end{abstract}

Objective: To compare the effects of packing with nonpacking of the perianal abscess cavity on patient discomfort and wound healing and subsidiary evaluation of the clinical outcomes.

Materials and Methods: This single centre randomized controlled trial was carried out on 50 patients who were operated upon for perianal abscess in Combined Military Hospital, Dhaka during the period of July 2014 to June 2016. Patients were randomly assigned to receive either packing or nonpacking treatment through allocation by sealed envelope. The packing (control) group was instructed to report to a single nursing staff for subsequent dressing with packing of the residual cavity. The nonpacking group was discharged with a superficial protective dressing; they did not undergo wound dressing but managed their own wounds until follow-up. Outcome measures were time of healing, abscess recurrence, fistula formation and post operative pain.

Results: A total of 54 patients were enrolled (4 lost in follow-up): 24 in the packing and 26 in the nonpacking arm. The two groups were comparable in terms of age and gender distribution, type, size of the abscess, duration of symptoms and length of follow-up. Healing in the non-packing group was faster compared to that of the packing group: mean 24.08 days versus 34.13 days $(P=0.000)$. The rate of abscess recurrence was similar $(P=0.664)$. Post operative fistula rates were similar $(P=0.623)$. Pain scores appeared less in the nonpacking arm and statistically significant (7.25 vs. $4.24, P=0.000)$.

Conclusion: Small size of the study population was the limitation of this study. In order to obtain a higher level of evidence, an adequately powered multicentre based prospective randomized controlled trial is required to definitely address the question of packing of the cavity and its beneficial outcome following incision and drainage in the management of perianal abscess.

Key-words: Perianal abscess, Non-packing, Packing, Anorectal disease.

\section{Introduction}

Anorectal abscess is a potentially debilitating condition and remains as one of the most common anorectal conditions encountered in practice ${ }^{1}$. The incidence of abscess is reportedly between 0.4 and $5 \%$ of patients undergoing operative management in USA ${ }^{2}$.

Anorectal abscesses are defined by the anatomic space in which they develop and are more common in the perianal and ischiorectal spacesand less common in the intersphincteric, supralevator and submucosal locations ${ }^{3}$. Perianal abscesses usually arise with indolent onset of constant, throbbing, acute anal pain associated with localized swelling, erythema and fluctuance. Perianal abscess can be readily discriminated from other causes of acute anal pain such as anal fissure and thrombosed external haemorrhoid by history and gentle visual inspection.

1. Col Md Tanvirul Islam, MBBS, FCPS (Surgery), Fellow Colorectal Surgery (NUH, Singapore), Classified Specialist in Surgery, Colon \& Rectal Surgeon, CMH, Dhaka 2. Brig Gen Md Mahbubur Rahman, MBBS, FCPS (Surgery), Fellow Colorectal Surgery (France), Advisor Specialist in Surgery (Colorectal) and Chief Surgeon, CMH, Dhaka 3. Lt Col Nasrin Begum, MBBS, MPhil, Assistant Professor of Anatomy, AFMC, Dhaka. 
Traditional management of perianal abscess involves early incision, drainage, curettage and packing of the residual cavity. The rationale for cavity dressings is initially to provide surgical haemostasis and then to prevent skin closure over the cavity, allowing healing by secondary intention ${ }^{4}$. Cavity packing and dressings, however, are problematic in that they cause significant discomfort and require multiple visits to change the packing until healing has occurred. There is currently no established evidence for this practice however; regular post operative packing can be painful ${ }^{5}$.

An alternative to the traditional approach is to perform adequate incision and drainage and then to allow healing without the use of cavity dressings ${ }^{4}$. The current guidance from The American Society of Colon and Rectal Surgeons suggests that with an adequately sized elliptical incision, post operative wound packing is usually not necessary ${ }^{1}$. In the UK, however, this has not been incorporated into standard practice, and in the absence of sufficient evidence that it is both safe and effective, most UK Surgeons continue to pack the cavity until healing is achieved, especially for perianal abscesses. There is currently no robust evidence to guide clinical practice. The aim, therefore, was to examine the effects of this approach on patient discomfort and wound healing with subsidiary evaluation of the clinical outcomes. A randomized, prospective trial was conducted to compare the conventional treatment of perianal abscess with that of incision and drainage without cavity dressings.

\section{Materials and Methods}

This was a single-centre randomized controlled trial. This study was carried out on 50 patients who were operated upon for perianal abscess in Combined Military Hospital (CMH), Dhaka. The recruitment period was from July 2014 to June 2016.

All patients, adults aged 20 years or above, presenting with anorectal abscess was included in this study. Exclusion criteria included patients under the age of 20 years or unwilling to give consent and associated other conditions like known fistulae, diabetes mellitus, crohn's disease, immune suppression, malignancy or other underlying causes. Patients with recurrent abscesses where the initial abscess drainage was considered inadequate (if the skin was not opened sufficiently to allow drainage of the abscess cavity) and those who underwent primary fistulotomy during drainage were also excluded from the study.
The patient recruitment was performed on first post operative day prior to any dressing change. Patients were randomly assigned to receive either packing or nonpacking through allocation by sealed envelope. An informed written consent was obtained from each patient willing to participate in the study.

Patients were treated with incision and drainage of the abscess cavity under general or spinal anaesthesia. The abscess was deroofed (involving an elliptical excision of perianal skin over the abscess cavity of sufficient length to drain the entire cavity and any extensions) to allow free drainage of the residual cavity. All patients were given a haemostatic pack intraoperatively, so the surgeon was blinded to the allocation of study group and had their dressing changed on first post operative day by the nursing staff.

On discharge, the packing (control) group were instructed to report to a single nursing staff of the hospital for subsequent dressing with packing of the residual cavity. Patients in the non-packing group were discharged with a superficial protective dressing to absorb any discharge from the cavity and protect the open wound. These patients were advised to have sitz bath, keep the area as clean and dry as possible. They did not undergo wound dressing but managed their own wounds until follow-up.

Data were collected on patient demographics, characteristics of the abscess (size and type), length of hospital stay, duration of symptoms before presentation, time for cavity healing, recurrent abscess or fistula formation, pain scores and length of follow-up. For the purposes of this study, abscesses were classified as superficial (superficial/ submucosal) or deep (ischiorectal/ intersphincteric). Pain scoring was achieved via a standard Visual Analog Scale (VAS) for pain, at the first outpatient visit,in which subjects were asked to score their average pain over the previous 2 weeks, rather than daily discomfort levels. The primary end-point was time for cavity healing. Secondary end-points were abscess recurrence, fistula formation and pain score at two week using a standard visual analogue scale (VAS).

The predefined follow-up period for each patient was until the abscess cavity had healed completely. The patients were then followed up for a minimum 6-month period for abscess recurrence or fistula formation. All patients were reviewed at two weekly intervals in the outpatient clinic until healing occurred. Healing was defined as the cavity being closed and the skin completely re-epithelialized. 
For patients who failed to attend the outpatient clinic, telephone interviews were conducted to determine time of healing (no longer requiring dressings, no further discharge), pain scores, and other data.

\section{Results}

Sixty patients presenting with perianal abscess were assessed for eligibility for the trial. Fifty-five patients were eligible for inclusion and 54 were enrolled. Patients were divided into the packing (control) group and the non-packing (intervention) group. Twenty-seven patients were allocated to each group. As block randomization was used, the end result involved equal patients in each group. Three subjects from packing group and one from non-packing group were lost in follow-up. All follow-up reviews were conducted in person. The flow of participants through each stage of the trial is described in Fig- 1 .

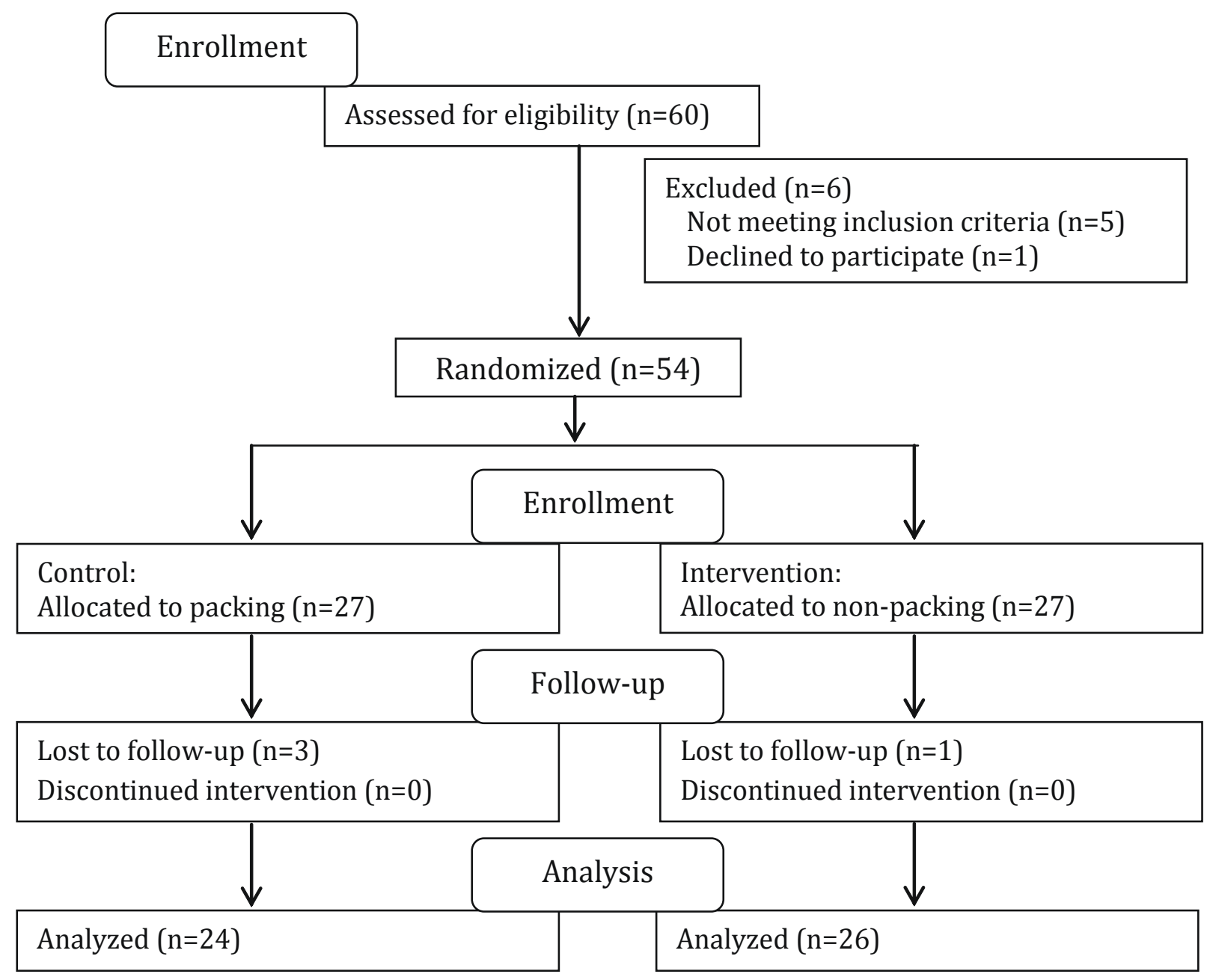

Fig-1: Flow of participants through each stage of the randomized trial

All continuous data were tested for normal distribution by nonparametric statistical method and analyzed accordingly using Mann-Whitney $U$ test. Fistula formation and abscess recurrence rate were analyzed using Fisher's exact probability test. Both tests were two-tailed with a significance level of 0.05 . Analysis was performed using SPSS statistical software version 19 (SPSS Inc. Chicago, IL, USA).

The two groups were comparable on a demographic basis with regards to age and gender distribution. Characteristics of the abscess in terms of type (superficial or deep), size, duration of symptoms before presentation and length of follow-up were also similar between the groups. Demographic data and abscess characteristics are outlined in Table-l. 
Table-I: Patient Demographics and Abscess Characteristics

\begin{tabular}{|l|c|c|c|c|}
\hline & & Packing $(\mathbf{n}=24)$ & Non-packing $(\mathbf{n = 2 6})$ & P value \\
\hline Age (years) & Median (Range) & $37.5(21-65)$ & $36.5(20-65)$ & 0.331 \\
\hline \multirow{2}{*}{ Gender } & Male & 19 & 20 & 0.850 \\
\cline { 2 - 4 } & Female & 05 & 06 & \\
\hline Abscess Type & Superficial & $16(66.7 \%)$ & $18(69.2 \%)$ & \multirow{2}{*}{0.848} \\
\cline { 2 - 4 } No (\%) & Deep & $8(33.3 \%)$ & $8(30.8 \%)$ & \\
\hline Median Abscess Size & (Range in mm) & $25(25-35)$ & $25(20-35)$ & 0.437 \\
\hline Median Duration of Symptoms & (Range in Days) & 02 & 03 & 0.061 \\
\hline Median Length of Follow-up & (Range in Week) & $4(3-24)$ & $3.5(14-90)$ & 0.773 \\
\hline
\end{tabular}

The mean time of healing (defined as complete epithelialization of the wound) was 34.13 (range, 16-110) days in the packing and 24.08 (range, 14-90) days in the non-packing group with significant $P$ value $(P=0.000)$. Post operative fistulas were detected at follow-up in eleven patients: six patients in the packing group (25\%), and five (19.2\%) in the non-packing group; this was not a significant difference $(P=0.623)$. There was also no difference in abscess recurrence rates between the groups (3/24 in packing group vs $2 / 26$ in non-packing group; $P=0.664)$. The non-packing group reported less pain at 2 weeks post operatively. At two weeks, the median pain score in the non-packing group was 4.25 compared with 7.25 in the packing group $(P=0.000)$. There were no differences in median length of stay $(P=0.709)$. No adverse or unexpected events were seen in either group. Results are summarized in Table-II.

Table-II: Post operative Outcome

\begin{tabular}{|l|c|c|c|}
\hline & Packing (n=24) & Non-packing (n=26) & P value \\
\hline Time of Healing Mean Days (Range) & $34.13(16-110)$ & $24.08(14-90)$ & 0.000 \\
\hline Fistula Formation & $6(25 \%)$ & $5(19.2 \%)$ & 0.623 \\
\hline Recurrent Abscess & $2(8.3 \%)$ & $2(7.7 \%)$ & 0.664 \\
\hline $\begin{array}{l}\text { Post Operative Pain Score at Two week } \\
\text { Median (Range) }\end{array}$ & $7.25(4-9)$ & $4.25(1-9)$ & 0.000 \\
\hline Length of Stay-Median Days(Range) & $1(1-2)$ & $1(1-2)$ & 0.609 \\
\hline
\end{tabular}

\section{Discussion}

Anorectal abscess is believed to originate from an infection in the anal glands. In 1880, Hermann and Desfosses demonstrated branching of the anal glands within the internal sphincter, submucosa and opening into the anal crypts. They were the first to suggest that infection in the anal glands results in extension of sepsis through the inter sphincteric space to the perianal tissues ${ }^{7}$. Tucker and Hellwing demonstrated definitively that anal sepsis originates in the anal ducts which allows the infection to extend from the anal lumen into the wall of the anal canal ${ }^{8}$.

The infection may extend between the internal and external sphincter, reach the anal verge to become a perianal abscess or it may rupture through the external sphincter and become an ischiorectal abscess. If the abscess extends cephalad in the rectal wall, a high intermuscular abscess will result and extension of abscess above the levators will produce a supralevator abscess. A deep postanal abscess may extend to either or both ischiorectal fossae resulting in a horseshoe abscess ${ }^{9}$.

The conventional management of perianal abscess often results in prolonged cavity dressings associated with increased cost, pain and inconvenience to patients with no evidence to support the practice. Alternative approaches to the surgical management of superficial abscesses have been investigated. Curettage and primary closure of the anorectal abscess cavity (30 cases) under antibiotic cover was first described by Ellis ${ }^{10}$ in 1951 who reported a high rate of primary healing (6.5 days). Ellis ${ }^{11}$ in 1960, reported a primary intention healing rate of 77 percent after primary suture of the curetted anorectal abscess cavity (151cases). Abraham et al ${ }^{12}$ reported a good result in terms of faster healing time and less time off work compared to packing of the abscess cavity. The primary healing rate was $78 \%$ but this approach was applied to soft tissue abscesses, perianal abscesses were not studied exclusively. Curetting of abscess cavities under antibiotic cover with primary closure by suturing, although advocated by Ellis ${ }^{10,11}$ and others ${ }^{12-14}$ this approach has failed to gain wide popular acceptance. 
De Pezzer catheter drainage is another alternative to traditional wound packing ${ }^{15}$. In 1990, Kyle and Isbister ${ }^{16}$ retrospectively compared the use of a de Pezzer catheter with traditional incision and drainage. The authors indicated that the de Pezzer catheter was well tolerated and compared favorably with the traditional technique ${ }^{16}$.

O'Malley et $\mathrm{al}^{5}$ undertook a randomized trial on the treatment of cutaneous abscesses (on the trunk, buttock and limbs) without cavity packing. Their results revealed that non packing did not cause any increased morbidity and the patients reported decreased pain scores. Koehleret al ${ }^{17}$ observed that cutaneous abscesses may be treated with incision and drainage alone thus avoiding pain and hospitalization. Kessler ${ }^{18}$ demonstrated in a randomized trial that wound packing does not impact failure or recurrence rates after simple incision and drainage, though there was no significant difference in pain scores. These information indicate that abscess cavities can be managed without the use of cavity dressings without an increase in complication rates. However, in many hospitals continued packing is recommended ${ }^{4}$.

Tonkin et $\mathrm{al}^{4}$ conducted a pilot study in 2004 comparing two groups of patients presenting with perianal abscesses treated by incision and drainage of the abscess cavity with and without packing. Their results demonstrated that mean healing times $(p=0.214)$, rate of abscess recurrence $(p=0.61)$, post operative fistula rates were similar $(P=0.38)$ and pain scores appeared much reduced in the non-packing arm, but did not attain statistical significance. They concluded that perianal abscesses can be managed safely without continued packing of the cavity. This study demonstrates reduced healing time and reduced post operative pain at 2 weeks. These findings were not demonstrated by Tonkinet $\mathrm{al}^{4}$. This study also found that packing did not confer any protection with respect to risk of subsequent abscess recurrence and fistula formation. Perera et $a^{6}{ }^{6}$ demonstrated that healing in the non-packing group was faster when compared to that of the packing group (mean 26.8 days, $\mathrm{P}=0.047$ ), the non-packing group reported less pain at 2 weeks post operatively $(P=0.030)$ and there were no differences in recurrence rates between the groups $(P=0.58)$. These findings are conforming to the present study.

A 2016 Cochrane database of systematic review demonstrated that it is unclear whether using internal dressings (packing) for the healing of perianal abscess cavities influences time to healing, wound pain, development of fistulae, abscess recurrence or other outcomes.
Despite this absence of evidence, the practice of packing of abscess cavities is common. Given the lack of high quality evidence, decisions to pack may be based on local practices or patient preferences. Further clinical research is needed to assess the effects and patients' experience of packing ${ }^{19}$.

\section{Conclusion}

This study demonstrates that perianal abscesses can be treated safely and effectively with incision and drainage of the abscess cavity alone with no requirement for continued cavity packing while packing of the abscess offered no protection with regards to abscess recurrence and fistula formation. The non-packing of anorectal abscess cavity is likely to provide significant savings in terms of nursing resources and to reduce the patient discomfort associated with frequent cavity dressing changes. The authors acknowledge that it's small population limits the study. In order to obtain a higher level of evidence, an adequately powered multicenter based prospective randomized controlled trial is required to definitely address the question if packing of the cavity is necessary or beneficial following incision and drainage in the management of perianal abscess.

\section{Acknowledgement}

We express our gratitude and full indebtedment to Brig Gen Md Ahsan Habib, Professor and Head, Department of Anatomy, Armed Forces Medical College, for helping us in data entry and data analysis by SPSS.

\section{References}

1. Steele SR, Kumar R, Feingold DL et al. Practice Parameters for the Management of Perianal Abscess and Fistula-in-Ano. Dis Colon Rectum 2011; 54:1465-74.

2. Davis BR, Kasten KR. Anorectal Abscess and Fistula. In: Steele SR, Hull TL, Read TE, Saclarides TJ, Senagore AJ, Whitlow CB, Editors. The ASCRS Textbook of Colon and Rectal Surgery. 3rd ed. New York: Springer; 2016:215-44.

3. Vogel JD, Johnson EK, Morris AM et al. Clinical Practice Guideline for the Management of Anorectal Abscess, Fistula-in-Ano and Rectovaginal Fistula. Dis Colon Rectum 2016; 59:1117-33.

4. Tonkin DM, Murphy E, Brooke-Smith M et al. Perianal abscess: A pilot study comparing packing with nonpacking of the abscess cavity. Dis Colon Rectum 2004; 47(9):1510-4.

5. O'Malley GF, Dominici P, Giraldo P et al. Routine packing of simple cutaneous abscesses is painful and probably unnecessary. Acad Emerg Med 2009; 16(5):470-3.

6. Perera AP, Howell AM, Sodergren MH et al. A pilot randomised controlled trial evaluating postoperative packing of the perianal abscess. Langenbecks Arch Surg 2015; 400:267-71. 
7. Hermann $G$, Desfosses L. Sur la muquese de la region cloacale de rectum. Compts Rend Acad Sci 1880; 90:1301-2(III).

8. Tucker CC, Hellwing CA. Histopathology of anal glands. Surg Gynecol Obstet 1933; 58:145-9.

9. Abcarian H. Anorectal infection: abscess-fistula. Clin Colon Rectal Surg 2011; 24:14-21.

10. Ellis M. The use of penicillin and sulphonamides in the treatment of suppuration. Lancet 1995; 1(6658):774-5.

11. Ellis M. Incision and primary suture of abscesses in the anal region. Proc $R$ Soc Med 1960; 53:652-4.

12. Abrahams N, Doudle M, Carson P. Open versus closed surgical treatment of abscesses: A controlled clinical trial. ANZ J Surg 1997; 67:173-6.

13. Leaper DJ, Page RE, Rosenberg IL et al. A controlled study comparing the conventional treatment of idiopathic anorectal abscess with that of incision, curettage and primary suture under systemic antibiotic cover. Dis Colon Rectum 1976; 19:46-50.
14. Barnes SM, Milsom PL. Abscesses: An open and shut case! Arch Emerg Med 1988; 5(4):200-5.

15. Isbister WH. A simple method for the management of anorectal abscess. ANZ J Surg 1987; 57:771-4.

16. Kyle S, Isbister WH. Management of anorectal abscesses: comparison between traditional incision and packing and de Pezzer catheter drainage. ANZ J Surg 1990; 60:129-31.

17. Koehler MB, Nakayama DK. Treatment of cutaneous abscesses without postoperative dressing changes. AORN J 2009; 90(4):569-74.

18. Kessler DO, Krantz A, Mojica M. Randomized trial comparing wound packing to no wound packing following incision and drainage of superficial skin abscesses in the pediatric emergency department. Pediatr Emerg Care 2012; 28(6):514-7.

19. Smith SR, Newton K, Smith JA et al. Internal dressings for healing perianal abscess cavities. Cochrane Database of Systematic Reviews 2016, Issue 8. Art No: CD011193. 